\title{
Propuesta de Un PROCEDIMIENTO PARA LA PLANEACIÓN DE LAS CAPACIDADES DE PRODUCCIÓN DE UNA EMPRESA
}

Ailen EsteVez-TorRes* https://orcid.org/0000-0002-4337-0250

Alexey Megna-Alicio https://orcid.org/0000-0001-6714-0452

RAFAEL EDUARDO JARDINES-RIVAS https://orcid.org/0000-0002-9771-6544

ISABEL CRISTINA PARRA-GARCÍA https://orcid.org/0000-0002-6623-5684

EduARDO LEÓN-PARRA https://orcid.org/0000-0001-8843-6025

GIANNY JIMENEZ-SILVA https://orcid.org/0000-0002-0561-2642 Universidad de Las Tunas, Cuba

Recibido: 28 de septiembre del 2020 / Aprobado: 23 de noviembre del 2020

doi: https://doi.org/10.26439/ing.ind2021.n40.4861

RESUMEN: La presente investigación tuvo como objetivo el diseño de un procedimiento para la planeación de las capacidades de producción de una empresa. Para su desarrollo se utilizaron los métodos de observación directa, histórico-lógico, hipotético-deductivo, análisis-síntesis, y las técnicas de observación, la revisión documental y encuestas. Por otro lado, se aplicó el método de expertos Delphi para validar el procedimiento. Se logró integrar aspectos positivos de ocho de los procedimientos consultados e incluir otros aspectos que no fueron abordados. Además, la planeación de las capacidades de producción se manifiesta como un proceso de mejora continua.

PALABRAS CLAVE: planeación de las capacidades de producción / planeación de la producción / mejora continua

\footnotetext{
*Correo electrónico: ailenet@ult.edu.cu
} 


\section{A PROPOSAL FOR A PROCEDURE TO PLAN THE PRODUCTION CAPACITY OF A COMPANY}

ABSTRACT: The objective of this research was to design a procedure to plan the production capacity of a company. In order to develop the research, the direct observation, historical-logical, hypothetico-deductive and analytic-synthetic methods, as well as observation techniques, document reviews and surveys, were used. In addition, the Delphi method was applied to validate the procedure. The integration of positive aspects from eight of the consulted procedures and the inclusion of other aspects not addressed in the research were achieved. Moreover, production capacity planning is shown as a continuous improvement process.

KEYWORDS: production capacity planning / production planning / continuous improvement 


\section{INTRODUCCIÓN}

El éxito en las organizaciones exige una continua adaptación de la empresa a su entorno, para lograr la máxima eficiencia en su funcionamiento interno. Es por esta situación dinámica de creciente complejidad, que las mismas se ven obligadas a apoyarse en nuevas herramientas y métodos que les permitan adaptarse a las condiciones cada vez más cambiantes de su medio. Una de las formas más efectivas de acoplarse a las realidades del ambiente es a través de técnicas de gestión.

La tendencia a la mejora de la organización del trabajo con fines de incrementar la productividad según Corrales Lima (2013) hace que se destaquen la gestión de la producción, dentro de la cual juega un papel fundamental la Organización Mundial del Trabajo.

Según Herrera Ochoa, Ramírez Castañeda y Mayorga Torres (2007), una proporción de empresas manufactureras (especialmente las pequeñas y medianas) desconocen la capacidad productiva teórica, instalada, disponible, necesaria y utilizada; esta es una debilidad organizacional y, especialmente, del proceso, ya que ignoran las potencialidades de los recursos, no determinan los cuellos de botella y no tienen base de datos para la toma de decisiones.

J. A. Domínguez Machuca, Álvarez Gil, M. Á. Domínguez Machuca, García González y Ruiz Jiménez (1995) y Urgal González y García Vázquez (2006) afirman que el modelo de planeación de las capacidades de producción en empresas productivas es una herramienta que valida este concepto y busca optimizar el proceso desde la planeación como respuesta organizacional a las exigencias del mercado.

Según plantea Estevez Torres (2019), la actividad productiva se ve afectada por las limitaciones impuestas por la capacidad disponible de los recursos máquina y por la mano de obra, es así como la formulación de la estrategia de ampliación de la capacidad, junto con los procesos de planeación y programación de las capacidades productivas se muestran como una de las temáticas más atractivas para la gestión de las operaciones.

Slack y Lewis (2017) plantean la gama de objetivos que se despliegan en los diferentes escenarios, dependiendo de la situación particular del sistema bajo estudio, e inclusive los propósitos particulares que presentan los subsistemas, orientados por diferentes planes, conllevan al desarrollo y formulación de un modelo para encaminar la planeación de capacidades.

Paredes Medina (1 de octubre de 2013) plantea que, si una empresa toma la decisión de hacer más de un producto o servicio, la planeación de la capacidad es la primera actividad que se debe llevar a cabo.

Chase Aquilano y Jacobs (2005) y J. A. Domínguez Machuca et al. (1995) establecen que la gestión de la capacidad de producción y las decisiones que conllevan a su desarrollo deben dirigirse desde el nivel directivo de la organización; otros como Slack y Lewis (2017) 
y Heizer y Render (2015) coinciden en que las decisiones de capacidad pueden analizarse desde tres niveles o tres horizontes de tiempo: corto plazo, mediano plazo y largo plazo.

La provincia de Las Tunas está ubicada en la región oriental de Cuba, la misma cuenta con catorce organizaciones que se dedican a la producción de bienes. En visitas a estas corporaciones se descubrió que hay un desconocimiento por parte de ellas mismas sobre la capacidad disponible que tienen, lo cual les impide asumir nuevos clientes, desarrollar nuevos productos y utilizar eficazmente la mano de obra, las materias primas y las instalaciones.

Autores como J. A. Domínguez Machuca et al. (1995); Acevedo Suárez, Gómez Acosta, Urquiaga Rodríguez y Hernández Torres (2002); Herrera Ochoa et al. (2007); Londoño Arboleda (2014); Igarza (2015); Oliva Gómez (2013); Urgal González y García Vázquez (2006) y Paredes Medina (1 de octubre de 2013) han contribuido al desarrollo de la planeación de las capacidades de producción, con pasos, metodologías y procedimientos. Tomando como referencia sus principales aportes y limitaciones, siguiendo las pautas del proceso de planificación del modelo de administración que plantea Luna González (2014) y la metodología de mejora continua o ciclo de Deming, el objetivo de la presente investigación se basa en diseñar un procedimiento para la planeación de las capacidades de producción en una empresa.

\section{MATERIALES Y MÉTODOS}

En el transcurso del proceso de investigación se visitaron catorce empresas ubicadas en la provincia de Las Tunas, Cuba, para conformar la población objeto de estudio, las cuales poseen diferentes características en cuanto a su sistema de producción e industria. A continuación, se muestra en la tabla 1 la composición por sectores de la población seleccionada. Están presentes en el estudio seis sectores industriales que se encuentran en desarrollo en el país.

Tabla 1

Composición de la población seleccionada

\begin{tabular}{lc}
\hline Clasificación de las empresas & Total \\
\hline Industria ligera & 3 \\
Industria pesada & 2 \\
Industria alimentaria & 4 \\
Agricultura & 2 \\
Construcción & 2 \\
Industria azucarera & 1 \\
\hline Total & 14 \\
\hline
\end{tabular}

Elaboración propia 
Posteriormente, se clasificó la población según el tipo de producción (homogénea o heterogénea), siguiendo los criterios que plantea Acevedo Suárez et al. (2002), lo cual puede verse en la tabla 2 .

Tabla 2

Clasificación del tipo de producción

\begin{tabular}{lc}
\hline Clasificación & Total \\
\hline Producciones homogéneas & 10 \\
Producciones heterogéneas & 4 \\
\hline
\end{tabular}

Elaboración propia

Por otro lado, utilizando la clasificación que plantean Acevedo Suárez et al. (2002) y Heizer y Render (2015), se clasificaron los sistemas de producción de cada una de las empresas estudiadas y los resultados se muestran en la tabla 3.

Tabla 3

Clasificación del sistema de producción de empresas estudiadas

\begin{tabular}{ccc}
\hline \multicolumn{3}{c}{ Relación productor-consumidor } \\
\hline $\begin{array}{c}\text { Contra almacén } \\
6\end{array}$ & $\begin{array}{c}\text { Entrega directa con cobertura en el ciclo } \\
\text { de entrega }\end{array}$ & $\begin{array}{c}\text { Entrega directa sin cobertura } \\
\text { en el ciclo de entrega }\end{array}$ \\
\hline Por ritmo & Forma de ejecutar la producción & 2 \\
\hline 5 & Programado & Por pedidos \\
\hline En masa & 5 & 4 \\
\hline 9 & Tipo de producción & Unitaria \\
\hline
\end{tabular}

Elaboración propia

Se determinó la población de 112 directivos implicados en el proceso de planeación de la producción. Se aplicaron encuestas a las 96 personas con un nivel de confianza del $99 \%$ y un margen de error del $5 \%$, entre ellos, directores, jefes de producción, jefes de turno, especialistas comerciales, jefes de personal, especialistas económicos e inversionistas; para conocer cómo se realizaba el proceso de planeación de las capacidades en sus respectivas organizaciones y saber sus consideraciones sobre los aspectos a tener en cuenta en dicho proceso. 
Teniendo en cuenta lo planteado por Chase et al. (2005) y J. A. Domínguez Machuca et al. (1995), quienes establecen que la gestión de la capacidad de producción y las decisiones que conllevan a su desarrollo deben promoverse en el nivel directivo de la organización.

El procedimiento diseñado tiene en cuenta la metodología del ciclo de dirección: planificar, organizar, dirigir y controlar que plantean Guzmán Campos y Motejo Quiros (6 de noviembre del 2013); Fernández León (12 de septiembre del 2013); Luna González (2014) y Thompson y Antezana (septiembre del 2006).

Este procedimiento solo abarca la fase de planificar, en la cual, según Luna González (2014), se analiza la situación actual de la empresa, se visualiza el futuro de la misma, determinándose los objetivos y recursos necesarios para alcanzar dichos objetivos, y se establece la estrategia de la empresa.

Además, se basa en la metodología de mejora continua o ciclo de Deming (Colorado, 2019), la cual plantea cuatro fases: planificar, hacer, verificar y actuar. En la fase planificar se tuvieron en cuenta las investigaciones realizadas por J. A. Domínguez Machuca et. al (1995) y Paredes Medina (1 de octubre de 2013) que plantean el análisis de la demanda, además, se incluyó el análisis de los proveedores lo cual fue resultado de las encuestas realizadas. En la fase hacer se determinaron las capacidades de producción según Acevedo Suárez et al. (2002) y Londoño Arboleda (2014). En la etapa verificar se definieron, según los resultados anteriores, la estrategia a llevar a cabo y las acciones para dar cumplimiento a la estrategia, para eso se tuvo en cuenta a Paredes Medina (1 de octubre de 2013). En la última etapa, que corresponde con la fase actuar del ciclo de Deming, se implementaron las acciones y se evaluaron los resultados.

Posteriormente, se aplicó el método Delphi para la validación del procedimiento diseñado. Es importante señalar que los diez expertos fueron seleccionados entre directivos, especialistas de producción de empresas del territorio y docentes especialistas en el tema.

Se encuestaron a los expertos para autoevaluar el nivel de conocimiento sobre el tema (ANC), se les solicitó que marcaran en una escala del 1 al 10 su autoevaluación al respecto. Y se determinó el coeficiente de conocimiento $(K c)$ como se muestra en la fórmula (I).

$$
K=0,5(K c+K a)
$$

Para determinar el coeficiente de argumentación de los especialistas (Ka), se tuvieron en cuenta elementos importantes como: su conocimiento acerca de la producción, sus 
años de experiencia, sus conocimientos teóricos y sus posibilidades de identificación de las fortalezas y debilidades de la planeación de la producción en cualquier industria.

Cada uno de los especialistas se autoevaluó estos aspectos, calificándose en tres niveles (alto, medio y bajo). Se ofrece el patrón de comparación utilizado para evaluar el coeficiente de argumentación de los expertos propuestos (Ka), donde se le atribuye a cada categoría una puntuación según su grado de importancia e influencia.

Con los valores obtenidos del coeficiente de conocimiento y el de argumentación, se calculó el coeficiente de competencia (K), utilizando la fórmula (II), con lo cual, finalmente, queda constituido el comité de expertos seleccionado.

$$
K=0,5(K c+K a)
$$

Para establecer y comparar los niveles de competencia de los expertos propuestos, se utilizó la siguiente escala: alta $(0,7<K<1,0)$, media $(0,5<K<0,7)$ y baja $(K<0,5)$. De acuerdo con este criterio, fueron seleccionados nueve expertos $(1,3,4,5,6,7,8,9,10)$, por poseer un alto nivel de competencia.

\section{RESULTADOS}

El procedimiento consta de cuatro etapas y diez pasos, los mismos se describen a continuación:

\subsection{Etapa 1. Evaluación del mercado}

- Paso 1. Análisis del mercado

El objetivo fundamental fue evaluar el comportamiento del mercado incluyendo los clientes, los proveedores y los competidores. Las técnicas a aplicar fueron: segmentación de mercado y estudios de mercado, encuestas, matriz de perfil competitivo de la empresa, matriz BCG (matriz de Boston Consulting Group) y matriz de Mckinsey.

- Paso 2. Análisis de la demanda

El objetivo fundamental fue conocer la demanda futura de un producto. Las técnicas a aplicar fueron el análisis de tendencias, las series históricas y las series temporales. 


\subsection{Etapa 2. Evaluación de la capacidad}

- Paso 3. Cálculo de las capacidades actuales

El objetivo fundamental fue calcular las capacidades actuales. Se podía aplicar el procedimiento propuesto por Acevedo Suárez et al. (2002) y Londoño Arboleda (2014), entre otros, sin embargo, dependía del tipo de producción que se realizara en la fábrica y sus respectivas características.

- Paso 4. Determinación del coeficiente de utilización de la capacidad

Para determinar este coeficiente se dividió la capacidad utilizada entre la capacidad disponible y se multiplicó por cien para expresarlo en porcentaje.

- Paso 5. Análisis de los factores que determinan la magnitud de la capacidad y su nivel de utilización

Para llevar a cabo este paso se tuvieron en cuenta los factores que propone J. A. Domínguez Machuca et al. (1995) y Acevedo Suárez et al. (2002). Para determinarlos se aplicaron las encuestas, la observación y el método Delphi.

\subsection{Etapa 3. Planeación}

- Paso 6. Aplicación de la matriz demanda-capacidad

Se realizó la matriz demanda-capacidad que no es más que el análisis de la expresión $\frac{\text { demanda }}{\text { capacidad }}$.

Si $\frac{\text { demanda }}{\text { capacidad }}<$ entonces estamos en la región I.

Si $\frac{\text { demanda }}{\text { capacidad }}>$ entonces estamos en la región II.

El estado ideal es en el cual $\frac{\text { demanda }}{\text { capacidad }}=1$.

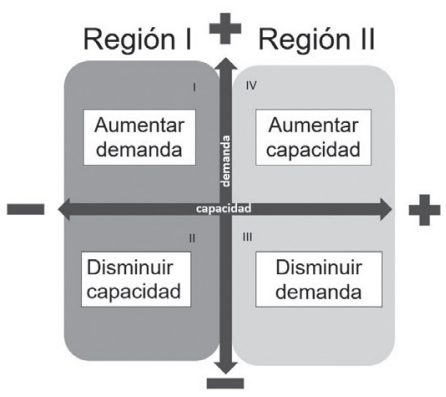

Figura 1. Matriz demanda-capacidad

Elaboración propia 
- Paso 7. Definición de la estrategia

En dependencia de la región en la cual se encontrará la matriz demanda-capacidad, y con el posicionamiento en el mercado, los proveedores, los clientes y el ciclo de vida del producto se decidía la estrategia; para ello se utilizó la matriz cuantitativa para valorar estrategias.

- Paso 8. Propuesta de acciones para dar cumplimiento a la estrategia

En este paso se propusieron las acciones de la estrategia, según el cuadrante identificado a partir de la relación demanda-capacidad. A cada región le corresponden dos cuadrantes, cada uno de los cuales tiene estrategias diferentes. En la tabla 4 se sugirieron las medidas generales en correspondencia con los resultados obtenidos en los pasos 6 y 7 .

Tabla 4

Medidas generales

Región I

\begin{tabular}{ll}
\hline & \multicolumn{1}{c}{ Región I } \\
\hline Cuadrante I & Reducir precios. \\
& Mejorar el mercadeo. \\
& Adaptarse al mercado a partir de cambios en los productos. \\
& Vender instalaciones, vender inventarios, despedir trabajadores o transferirlos. \\
& Guardar instalaciones o colocarlas en estado de reserva. \\
Cuadrante II & Desarrollar e introducir nuevos productos. \\
\hline & Aumentar el precio. \\
\hline & Programar largos períodos de entrega. \\
\hline & Dejar de estimular negocios con beneficios marginales. \\
& Adquirir nuevas instalaciones, equipos o mano de obra. \\
& Expandir las instalaciones existentes o reactivar alguna en estado de reserva. \\
& Analizar los cuellos de botella que limitan las capacidades y decidir sobre su \\
& eliminación o mejoramiento. \\
\hline
\end{tabular}

Fuente: Estevez Torres (2019)

\subsection{Etapa IV. Implementación}

- Paso 9. Implementación de las acciones

Se llevaron a cabo las medidas planteadas en el paso anterior; la aplicación de estas acciones quedó por parte de la empresa. 
- Paso 10. Evaluación de los resultados

Se evaluaron los resultados obtenidos y de ser necesario se volvía al paso 1 para analizar nuevamente el mercado o al paso 8 para proyectar nuevas soluciones en caso de que las adoptadas no cumplieran su objetivo.

A modo resumen, la calidad de la planificación de las capacidades quedó determinada por la aplicación sistemática del procedimiento; se recomienda la realización de un estudio de este tipo una vez al año, para perfeccionar la planificación.

A continuación, en la figura 2 se muestra el esquema del procedimiento diseñado.

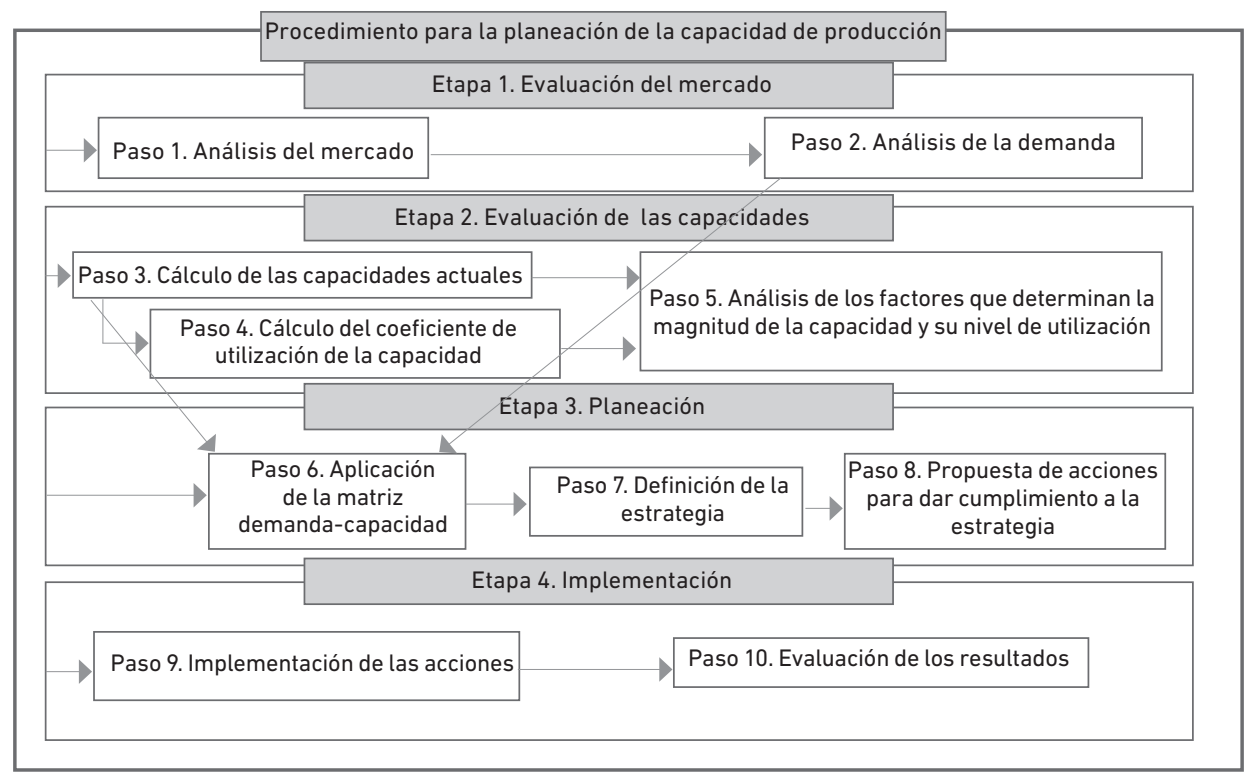

Figura 2. Diseño del procedimiento para la planeación de las capacidades de producción

Elaboración propia

\subsection{Validación del procedimiento mediante método de expertos}

Para evaluar la viabilidad de la aplicación del procedimiento propuesto, se aplicó una encuesta a los expertos donde se obtuvo el siguiente resultado:

- $\quad$ El $100 \%$ cree que es necesario la aplicación del procedimiento diseñado.

- El $90 \%$ considera que el procedimiento diseñado se adapta a las características de cualquier empresa productiva.

- El 95 \% plantea que existe una secuencia lógica de las etapas, las fases y los pasos. 
- Los expertos coinciden $100 \%$ que facilitaría el proceso de toma de decisiones, contribuiría a minimizar las deficiencias en la planeación de la producción y que tiene grandes posibilidades de generalización.

\section{DISCUSIÓN}

Igarza (2015) propone una metodología para la planificación de la producción en el taller de estructuras metálicas, en la empresa METUNAS; también Oliva Gómez (2013) propone un procedimiento para determinar la capacidad productiva en la línea de muebles de madera para la producción de sillas de restaurante \#1 en la empresa de muebles y lámparas, LUDEMA, de Las Tunas, ambos desarrollaron sus investigaciones en las empresas objeto de estudio, pero por ser empresa con producciones unitarias y que sus procedimientos solo contemplan un solo tipo de surtido no pudieron adaptarse a otras producciones.

En el caso de Acevedo Suárez et al. (2002), propone un procedimiento para el cálculo, balance y análisis de las capacidades de producción de una empresa, pero no contempla herramientas de planificación ni de gestión.

J. A. Domínguez Machuca et al. (1995) plantea un procedimiento, pero no toma en cuenta la planificación como un proceso continuo.

El procedimiento diseñado constituye una poderosa herramienta de planificación. Garantiza que se tenga en cuenta el mercado y el análisis de la demanda para proyectar la capacidad y tomar medidas al respecto.

La aplicación de este procedimiento, además, evita perder nichos del mercado a la empresa. Tiene en cuenta el análisis de los proveedores, aspecto que no está incluido en ninguno de los procedimientos analizados. Esto permite garantizar que el nivel de inventario se mantenga en niveles óptimos en todo momento, es decir, que no haya exceso o falta de existencias.

Por otro lado, garantiza que se logre una utilización óptima de la capacidad de producción, mediante una programación adecuada de los elementos de la máquina, lo que reduce el tiempo de inactividad y el exceso de uso. También garantiza que el tiempo de producción se mantenga en un nivel óptimo y, por lo tanto, aumente la productividad.

En el procedimiento se establece la planeación de las capacidades de producción como un proceso sistemático y en mejora continua al estar en plena correspondencia con el ciclo de Deming. 


\section{CONCLUSIONES}

La sistematización de los referentes teóricos permitió establecer las bases de la planeación de las capacidades de producción y su importancia en el desarrollo de una empresa.

Se logró integrar aspectos positivos de ocho de los procedimientos consultados e incluir otros aspectos que no fueron abordados por los mismos.

El diseño del procedimiento para la planeación de las capacidades de producción permitió obtener una herramienta precisa para contribuir a la planeación de la producción. El mismo es aplicable a empresas con producciones homogéneas y heterogéneas.

El procedimiento, además, está en plena correspondencia con el ciclo de Deming o el ciclo de mejora continua, aspecto que no ha sido abordado en ninguno de los procedimientos consultados.

\section{REFERENCIAS}

Acevedo Suárez, J. A., Gómez Acosta, M. I., Urquiaga Rodríguez, A. J., y Hernández Torres, M. (2002). Organización de la producción y los servicios. La Habana: Ediciones CUJAE.

Chase, R. B., Aquilano, N. J., y Jacobs, F. R. (2005) Administración de producción y operaciones para una ventaja competitiva. México D. F.: McGraw-Hill.

Colorado, F. (2019). El ciclo PHVA de Deming y el proceso administrativo de Fayol. Recuperado de https://www.academia.edu/5110051/3_Articulo_El_Ciclo_PHVA_de_ Deming_y_al_Proceso_Administrativo_de_Fayol

Corrales Lima, Y. X. (2013). Estudio de organización del trabajo en el taller de geomática de la Agencia Geocuba Las Tunas (tesis de grado). Universidad de Las Tunas, Cuba. Recuperada de http://hdl.handle.net/123456789/1732

Domínguez Machuca, J. A., Álvarez Gil, M. J., Domínguez Machuca, M. Á., García González, S., y Ruiz Jiménez, A. (1995). Dirección de operaciones. Aspectos estratégicos en la producción y los servicios. Madrid: McGraw-Hill.

Estevez Torres, A. (2019). Procedimiento para la planeación de las capacidades de producción. Caso de estudio muebles de habitaciones para el turismo en la UEB Muebles Ludema (tesis de maestría). Universidad de Las Tunas, Cuba. Recuperado de http://hdl.handle.net/123456789/4039

Fernández León, 0. (12 de septiembre del 2013) Planificar, organizar, liderar y controlar o el arte de gestionar nuestros despachos. Legal Today. Recuperado de https://www.legaltoday.com/opinion/blogs/gestion-del-despacho-blogs/ 
blog-manual-interno-de-gestion/planificar-organizar-liderar-y-controlar-oel-arte-de-gestionar-nuestros-despachos-2013-09-12/

Guzmán Campos, A. E., y Motejo Quiros, A. M. (6 de noviembre del 2013). El proceso administrativo según varios autores. Gestiopolis. Recuperado de https://www. gestiopolis.com/el-proceso-administrativo-segun-varios-autores/

Heizer, J., y Render, B. (2015). Dirección de la producción. Decisiones estratégicas. Madrid: Pearson Educación.

Herrera Ochoa, O. J., Ramírez Castañeda, L. N., y Mayorga Torres, O. (2007). Aplicación del modelo de planeación de las capacidades productivas en empresas manufactureras en una pyme del sector calzado. Memorias, Revista Nacional de Investigaciones, 5(9), 28.

Igarza, Y. R. (2015). Propuesta de una metodología para la planificación de la producción en el taller de estructuras metálicas de la empresa METUNAS (tesis de grado). Universidad de Las Tunas, Cuba.

Londoño Arboleda, M. F. (2014). Planeación de la capacidad de producción para la nueva fábrica de muebles de la empresa Iván Botero Gómez S. A. (tesis de grado). Universidad Nacional de Colombia, Manizales, Colombia. Recuperada de https://repositorio.unal.edu.co/bitstream/handle/unal/52715/9729922.2014. pdf?sequence=1\&isAllowed $=y$

Luna González, C. A. (2014). Proceso administrativo. México D. F.: Grupo Editorial Patria.

Oliva Gómez, A. I. (2013). Propuesta de procedimiento para determinar la capacidad productiva en la línea de muebles de madera para la producción de sillas de restaurante \#1 en la Empresa de Muebles y Lámparas (LUDEMA) de Las Tunas (tesis de grado). Universidad de Las Tunas, Cuba.

Paredes Medina, W. F. (1 de octubre del 2013). Capacidad del sistema de producción, conceptos generales. Gestiopolis. Recuperado de https://www.gestiopolis.com/ capacidad-del-sistema-de-produccion-conceptos-generales/

Slack, N., y Lewis, M. (2017). Operations Strategy (4. ${ }^{a}$ ed.). Londres: Pearson.

Thompson B., J. M., y Antezana, J. (septiembre de 2006). El proceso administrativo. Promonegocios.net. Recuperado de https://www.promonegocios.net/administracion/proceso-administrativo.html

Urgal González, B., y García Vázquez, J. M. (2006). Decisiones de producción, capacidades de producción y prioridades competitivas: Un estudio aplicado al sector de metal en España. Investigaciones Europeas de Dirección y Economía de la Empresa, 12(3), 133-149. 